POS PROCEEDINGS

\title{
The Perugia Variability Monitoring of Blazars
}

\section{Stefano Ciprini}

1. Physics Department, University of Perugia, via A. Pascoli, 06123 Perugia, Italy

2. I.N.F.N. Section of Perugia, via A. Pascoli, 06123 Perugia, Italy

3. Tuorla Observatory, Department of Physics and Astronomy, University of Turku, Väisäläntie 20, 21500 Piikkiö, Finland

E-mail: stefano.cipriniepg.infn.it

\section{Gino Tosti}

1. Physics Department, University of Perugia, via A. Pascoli, 06123 Perugia, Italy

2. I.N.F.N. Section of Perugia, via A. Pascoli, 06123 Perugia, Italy

\section{Giuliano Nucciarelli}

Physics Department, University of Perugia, via A. Pascoli, 06123 Perugia, Italy

Marco Bagaglia, Massimo Fiorucci*, Domenico Impiombato, Melissa Luciani*, Paolo Maffei*, Nicola Marchili*, Sergio Pascolini*, Vincenzo Picarelli*, Nicola Rizzi*, Fabio Roncella*

Physics Department, University of Perugia, via A. Pascoli, 06123 Perugia, Italy

\begin{abstract}
In fifteen years of optical monitoring performed with the $0.4 \mathrm{~m}$ automatic imaging telescope (AIT) of the Perugia University Observatory, more than 35000 VRI (and a few B) photometric data points were collected, contributing to get an important database of the multi-band optical history of 37 blazars brighter than V-mag 17. This blazar variability monitoring started in 1991, with a fully robotic implementation operational from 1994. Despite of the limited budget, manpower and telescope size, such long-term program allowed to obtain light curves of blazars with a rather unprecedented continuity and sampling for about a dozen of our targets (considering data usually obtained by a single telescope). This effort was very useful to trigger observations and campaigns at other frequencies, to study the optical variability modes and the temporal behavior on intermediate and long timescales. Some highlights and examples of results are briefly reported.
\end{abstract}

Workshop on Blazar Variability across the Electromagnetic Spectrum April 22-25, 2008

Palaiseau, France

\footnotetext{
*Formerly affiliated.
} 


\section{Introduction}

The rapid and violent optical variability is one of the defining properties of blazars. Detailed observations and study of the blazar flux variations can provide a considerable amount of information on the nature, structure, location and dynamics of the emitting regions. The knowledge of the temporal behavior on different timescales allow to study characteristic timescales, fluctuation modes, flare shapes and amplitudes, duty cycles and spectral changes. Moreover the knowledge of the optical variability behavior is important to understand the significance of the correlations with the fluctuations observed in other spectral bands. In particular, the so-called low/intermediatefrequency peaked BL Lac objects (LBL/IBL) show the peak of the synchrotron emission around IR-optical wavelengths, and displays usually large amplitude flares with prominent flux variations in a wide range of timescales. A multi-band, possibly well sampled and long-term optical monitoring is an important element of the multiwavelength analysis.

Unfortunately, the optical data collected in the past, although useful, are not suitable to perform a quantitative analysis of variability. These data, in fact, were collected either during episodic campaigns on a single source with typical duration of a few week, or during long term monitoring programs carried out on many sources but with a quite low sampling rate on the individual source (10 photometric measures per year, typically). For this reason there are still many open questions about blazar variability. In the recent years blazar monitoring programs designed on small-middle size (possibly automatic) telescopes, and international consortiums and campaigns, have increased the amount of optical data and the quality of monitoring (for example see [10, 22, 11, 1, 14]). Here we report a summary about one of the first automatic telescopes in the world devoted (from 1991) to a long-term optical photometric monitoring of a sample of bright northern blazars.

\section{The Perugia Telescope}

The Automatic Imaging Telescope (AIT) of the Perugia University Observatory ${ }^{1}$ (Est Longitude $00 \mathrm{~h} 49 \mathrm{~m} 34 \mathrm{~s}$; Latitude $+43^{\circ} 06^{\prime} 44^{\prime \prime}$ ) is based on an existing telescope transformed into an
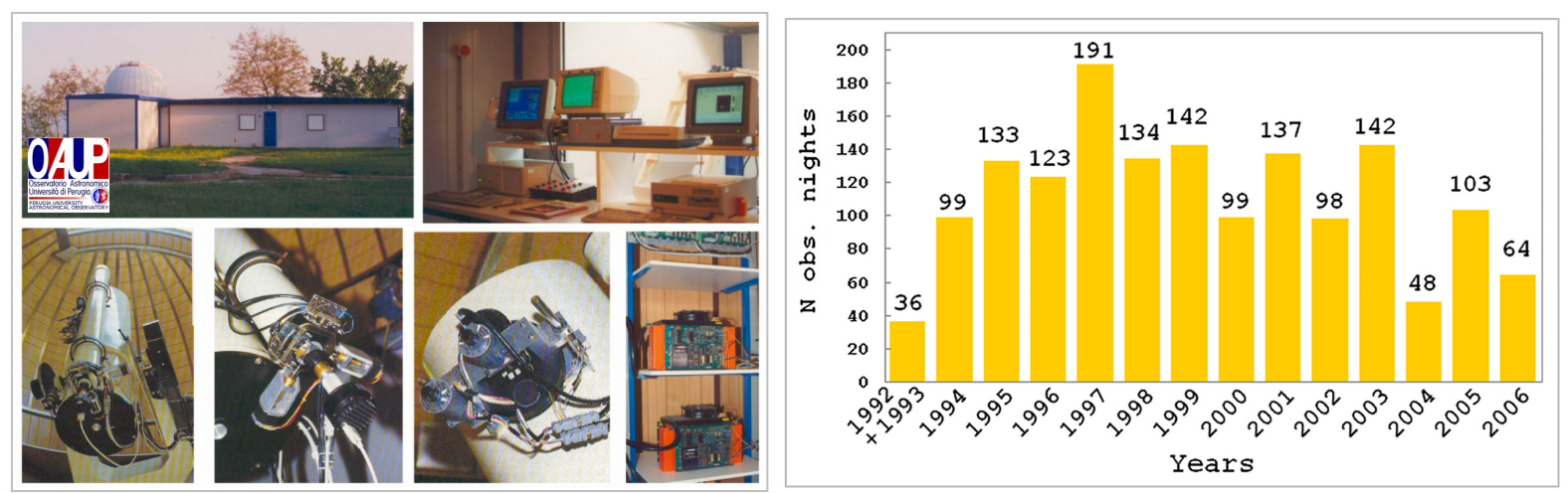

Figure 1: Left panel: Some views of the Automatic Imaging Telescope (AIT) of the Perugia University Observatory and its instrumentation. This $40 \mathrm{~cm}$ telescope started the observations in 1991, with fully robotic operations from 1994 (being therefore one of the first in the world). The telescope is mainly dedicated to $V R I$ variability monitoring of bright $(V<17)$ blazars, through automatic and unattended operations. Right panel: The yearly number of observing nights with validated photometric data, obtained by the AIT.

\footnotetext{
${ }^{1}$ http://astro.fisica.unipg.it
} 

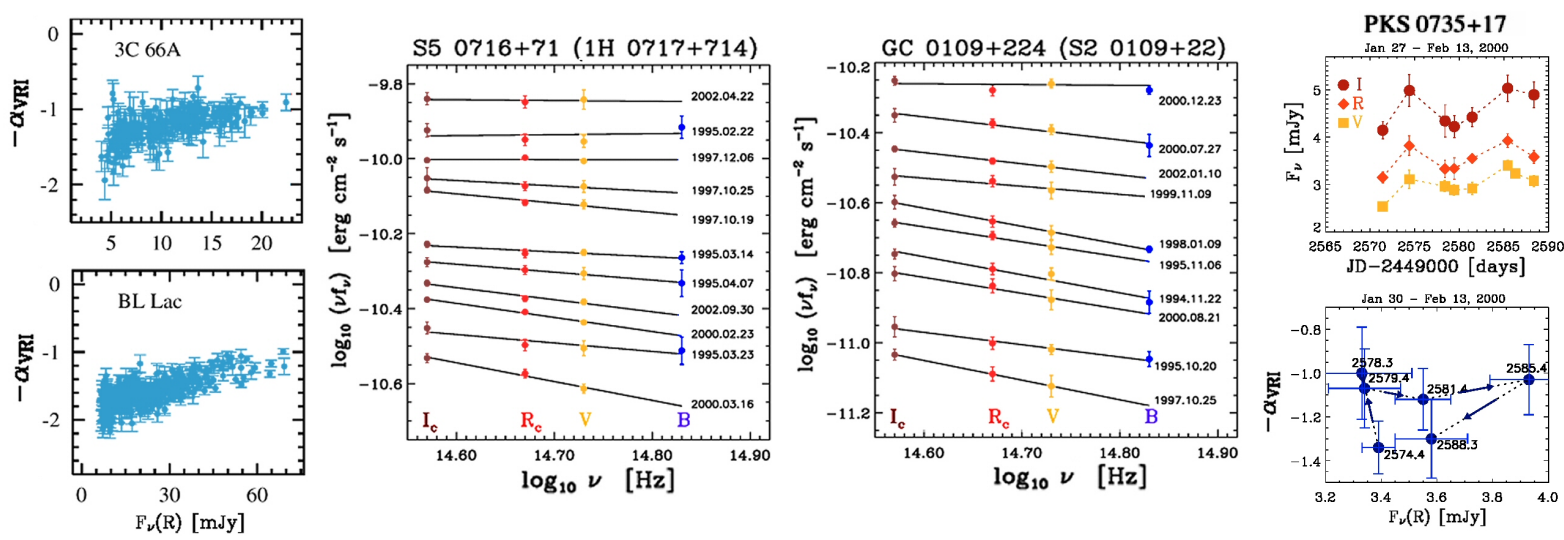

Figure 2: Left panels: dependence of the VRI-flux spectral slope on the flux intensity for the blazars 3C 66A, and BL Lac. The scattering (only partially explainable as statistical fluctuations) is evident. From the analysis of our entire database results that the more extremely variable blazars show a general trend that flattens the slope when the source is brighter. Central panels: the $B V R I$ and $V R I$ spectral energy distribution of the blazars GC 0109+224 and S5 0716+71, with a selection of different optical continuum spectra. Right panels: the evolution of the optical spectrum of PKS $0735+178$ as a function of the flux in the $R$ band during a moderate flaring activity. A clockwise loop is hinted. Adapted from [6, 3].

automatic instrument (Fig.1). Observations started in 1991, with the automatic implementation inaugurated in October 1994. AIT is a 40-cm Newtonian reflector (f/5) with an equatorial mount, and two brushless motors controlled by a two axis motor controller for the slewing. All the observatory functions are controlled by two PCs in unattended mode during the night. The system is equipped with an auto-guider, and the software is designed to allow the automatic reduction of the photometry data at the end of each night. For a detailed description of the telescope, hardware and software systems see [21]).

\section{The Blazar Monitoring Program}

AIT is almost entirely dedicated to the automatic optical monitoring of blazars ${ }^{2}$. Since the end of 1991 more than 35000 final photometric Johnson $B$ (only a few), $V$ and Cousins $R$ and $I$ are collected for about 40 blazars. In Fig. 3 is reported the list of our best monitored 37 blazars, with some basic properties. In particular, for a dozen of well-known blazars, we achieved unprecedented monitoring results (observing continuity and total number of final data points) for a single instrument.

The exposure times are in the range of 3-8 min, and the duration of an observing run is less than 1 hour for each target. CCDs frames are processed by an automatic procedure performing dark current and bias correction, field recognition, star finding, evaluation of comparison stars, and the calculation of the instrumental magnitudes with the method of aperture photometry (typical aperture radius is 4 arcsec). The standard Johnson-Cousins magnitudes are then obtained using our own calibrated sequences of comparison stars in the same field of the blazar. More details on the procedures, software, photometric system adopted, the calibration of new comparison stars sequences, as well as early results are reported for example in $[21,8,9,20,7,17,16]$. Moreover detailed analysis and results on a decade of our optical multi-band observations of single sources,

\footnotetext{
${ }^{2}$ Sometimes short observing runs were devoted also to the variability monitoring on dwarf novae, see e.g. [13]
} 
The Perugia Variability Monitoring of Blazars

\begin{tabular}{|c|c|c|c|c|c|c|c|}
\hline \multirow{2}{*}{$\begin{array}{l}\text { IAU name } \\
\text { (B1950) }\end{array}$} & \multirow[t]{2}{*}{ Common name } & \multicolumn{2}{|c|}{ Coordinates (J2000) } & \multirow[t]{2}{*}{$z$} & \multirow[t]{2}{*}{$A_{\mathrm{v}}$} & \multirow[t]{2}{*}{$\alpha_{\mathrm{RX}}$} & \multirow[t]{2}{*}{ Class } \\
\hline & & $\alpha$ & $\delta$ & & & & \\
\hline $0109+224$ & GC0109+224 & 011205.8 & +224439 & $>0.23$ & 0.124 & 0.86 & LBL \\
\hline $0219+428$ & $3 \mathrm{C} 66 \mathrm{~A}$ & 022239.6 & +430208 & 0.444 & 0.279 & $0.78^{ \pm 0.07}$ & LBL \\
\hline $0235+164$ & $\mathrm{AO} 0235+164$ & 023838.9 & +163700 & 0.940 & 0.265 & $0.89^{ \pm 0.07}$ & LBL \\
\hline $0300+470$ & 4C 47.08 & 030335.2 & +471617 & & 0.870 & 0.92 & LBL \\
\hline $0316+413$ & NGC 1275 & 031948.2 & +413042 & 0.017 & 0.540 & 0.84 & \\
\hline $0323+022$ & H $0323+022$ & 032613.9 & +022514 & 0.147 & 0.372 & $0.55^{ \pm 0.07}$ & HBL \\
\hline $0414+009$ & 2E $0414+0057$ & 041652.4 & +010524 & 0.287 & 0.393 & $0.54^{ \pm 0.01}$ & HBL \\
\hline $0422+004$ & PKS 0422+00 & 042446.8 & +003607 & $>0.31$ & 0.335 & 0.87 & LBL \\
\hline $0716+714$ & S5 0716+71 & 072153.3 & +712036 & $>0.30$ & 0.102 & $0.82^{ \pm 0.03}$ & LBL \\
\hline $0735+178$ & PKS $0735+17$ & 073807.4 & +174219 & $>0.42$ & 0.116 & $0.92^{ \pm 0.02}$ & LBL \\
\hline $0754+100$ & PKS $0754+100$ & 075706.7 & +095635 & 0.280 & 0.075 & $0.84^{ \pm 0.02}$ & LBL \\
\hline $0806+524$ & 1ES $0806+524$ & 080949.2 & +521858 & 0.138 & 0.147 & 0.59 & HBL \\
\hline $0829+046$ & PKS 0829+046 & 083148.9 & +042939 & 0.180 & 0.108 & $0.86^{ \pm 0.01}$ & LBL \\
\hline $0851+202$ & OJ 287 & 085448.8 & +200630 & 0.306 & 0.094 & $0.81^{ \pm 0.03}$ & LBL \\
\hline $0912+297$ & Ton 396 & 091552.4 & +293324 & & 0.081 & $0.71^{ \pm 0.02}$ & HBL \\
\hline $0954+658$ & S4 0954+65 & 095847.2 & +653354 & 0.367 & 0.380 & $0.90^{ \pm 0.01}$ & LBL \\
\hline $1101+384$ & Mrk 421 & 110427.2 & +381232 & 0.031 & 0.051 & $0.56^{ \pm 0.03}$ & HBL \\
\hline $1147+245$ & OM 280 & 115019.2 & +241754 & $>0.20$ & 0.090 & $0.92^{ \pm 0.06}$ & LBL \\
\hline $1215+303$ & ON 325 & 121752.1 & +300701 & 0.130 & 0.079 & $0.75^{ \pm 0.02}$ & HBL \\
\hline $1219+285$ & W Com & 122131.7 & +281358 & 0.102 & 0.075 & $0.81^{ \pm 0.07}$ & LBL \\
\hline $1226+023$ & $3 C 273$ & 122906.7 & +020308 & 0.158 & 0.068 & $0.85^{ \pm 0.10}$ & FSQ \\
\hline $1253-055$ & $3 C 279$ & 125611.1 & -054721 & 0.538 & 0.095 & $0.93^{ \pm 0.03}$ & FSQ \\
\hline $1415+259$ & $2 \mathrm{E} 1415+2557$ & 141756.6 & +254325 & 0.237 & 0.062 & $0.55^{ \pm 0.02}$ & HBL \\
\hline $1418+546$ & OQ 530 & 141946.6 & +542314 & 0.152 & 0.044 & $0.87^{ \pm 0.02}$ & LBL \\
\hline $1424+240$ & PKS $1424+240$ & 142700.5 & +234800 & & 0.194 & $0.70^{ \pm 0.01}$ & HBL \\
\hline $1458+228$ & MS $14588+2249$ & 150101.9 & +223806 & 0.235 & 0.159 & $0.59^{ \pm 0.12}$ & HBL \\
\hline $1611+343$ & OS 319 & 161341.0 & +341248 & 1.404 & 0.060 & 0.92 & FSQ \\
\hline $1641+399$ & $3 C 345$ & 164258.8 & +394837 & 0.594 & 0.044 & $0.91^{ \pm 0.01}$ & FSQ \\
\hline $1652+398$ & Mrk 501 & 165352.2 & +394536 & 0.033 & 0.064 & $0.63^{ \pm 0.05}$ & HBL \\
\hline $1722+119$ & H $1722+119$ & 172504.4 & +115216 & 0.159 & 0.568 & $0.50^{ \pm 0.08}$ & HBL \\
\hline $1727+502$ & I Zw 187 & 172818.6 & +501311 & 0.055 & 0.098 & $0.61^{ \pm 0.02}$ & HBL \\
\hline $1807+698$ & $3 C 371$ & 180650.7 & +694928 & 0.050 & 0.119 & $0.83^{ \pm 0.06}$ & LBL \\
\hline $2032+107$ & PKS 2032+107 & 203522.0 & +105606 & 0.601 & 0.474 & $0.80^{ \pm 0.08}$ & LBL \\
\hline $2200+420$ & BL Lac & 220243.3 & +421639 & 0.069 & 1.091 & $0.84^{ \pm 0.04}$ & LBL \\
\hline $2251+158$ & $3 \mathrm{C} 454.3$ & 225357.7 & +160854 & 0.859 & 0.355 & $0.89^{ \pm 0.01}$ & FSQ \\
\hline $2254+074$ & PKS 2254+074 & 225717.3 & +074312 & 0.190 & 0.219 & $0.91^{ \pm 0.03}$ & LBL \\
\hline $2344+514$ & 1ES $2344+514$ & 234704.8 & +514218 & 0.044 & 0.716 & $0.60^{ \pm 0.02}$ & HBL \\
\hline
\end{tabular}

Figure 3: List of 37 blazars monitored at the Perugia University Observatory with the AIT, during the observing program started in 1991. Columns contain names, positions, redshifts, interstellar absorption coefficients, radio-to-X-ray spectral indexes and the blazar class: LBL (low-energy peaked BL Lacs); HBL (high-energy peaked BL Lacs), FSQ (flat spectrum radio quasars). Adapted from [6].

or on data on flaring periods of single sources (like GC 0109+224, S5 0716+71, Mrk 421, W Com, BL Lac) can be found for example in $[18,19,15,5,4,3]$.

In Fig.4,5 and 6 the $V R I$ light curves collected during our monitoring program on sixth of our best sampled targets (GC 0109+224, NGC 1275, S5 0716+71, W Com, BL Lac, 3C 454.3) are reported as example.

\section{Optical spectral indexes}

Our multi-band (mostly $V R I$ ) simultaneous data allow to analyze the optical dereddened spectral flux distribution. Usign our database we found that LBLs show a spectral slope of 1.5, as expected in some SSC models [2], while the optical emission of HBLs and FSRQs is probably contaminated by other components at optical waveleghts. In fact sometimes high photometric measures can be complicated by the presence of a strong host galaxy component, which adds un- 

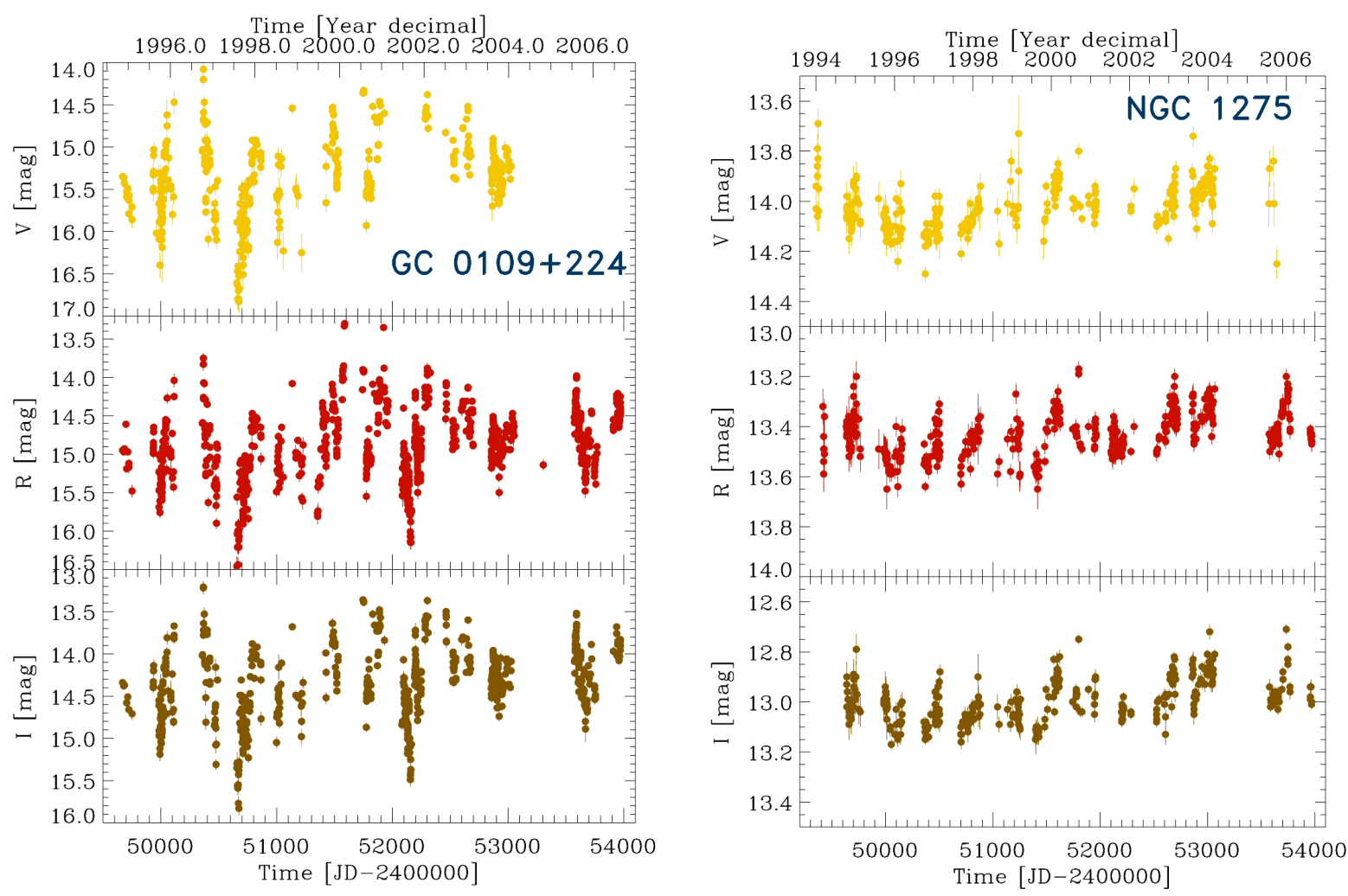

Figure 4: Optical VRI light curves of GC 0109+224 (1940 data points collected between Nov.1994 and Aug.2006), and of NGC 1275 (1252 data points collected between Dec.1993 and Aug.2006), obtained by the AIT.

wanted flux to the measurement and introduces a seeing-dependence to the flux that can plaque micro-variability studies [12].

From the analysis of our entire database results that the more extremely variable blazars show a general trend that flattens the slope when the source is brighter (Fig.2). During sufficiently sampled flares, we found evidence of characteristic loop-like variability patterns in the spectral index versus the flux plane. This behavior, usually seen in X-ray bands, implies changes in the injection of accelerated particles in the flaring blobs of the jet also at optical energy bands [5, 3]. For more results on the multi-band spectral analysis of our database see [6].

\section{Optical light curves}

Our blazars (especially LBLs) show in the optical band large amplitude flares, outbursts and brightening trend phases covering a duration range from hours to years (Fig.4, 5, 6). Four optical variability modes can be tentatively identified. 1) intermittent variability: characterized by a not regular alternation of semi-quiescent and bursting phases, with fine temporal structure of bursts showing relevant IDV, like in BL Lac. 2) quasi-regular variability: characterized by the occurrence of long term trends superposed to small scale faster fluctuations, like in 3C 273. 3) mixed variability: characterized by semi-regular long term trends, showing occasionally large isolated bursts, like in W Com. 4) pseudo-cyclic variability: characterized by recurrent outburst and oscillating trends, that could be related to geometrical/orientation effects, like in OJ 287. Several mid-term (i.e. days-weeks) characteristic time scales can be identified usually in our optical data (see, e.g. 

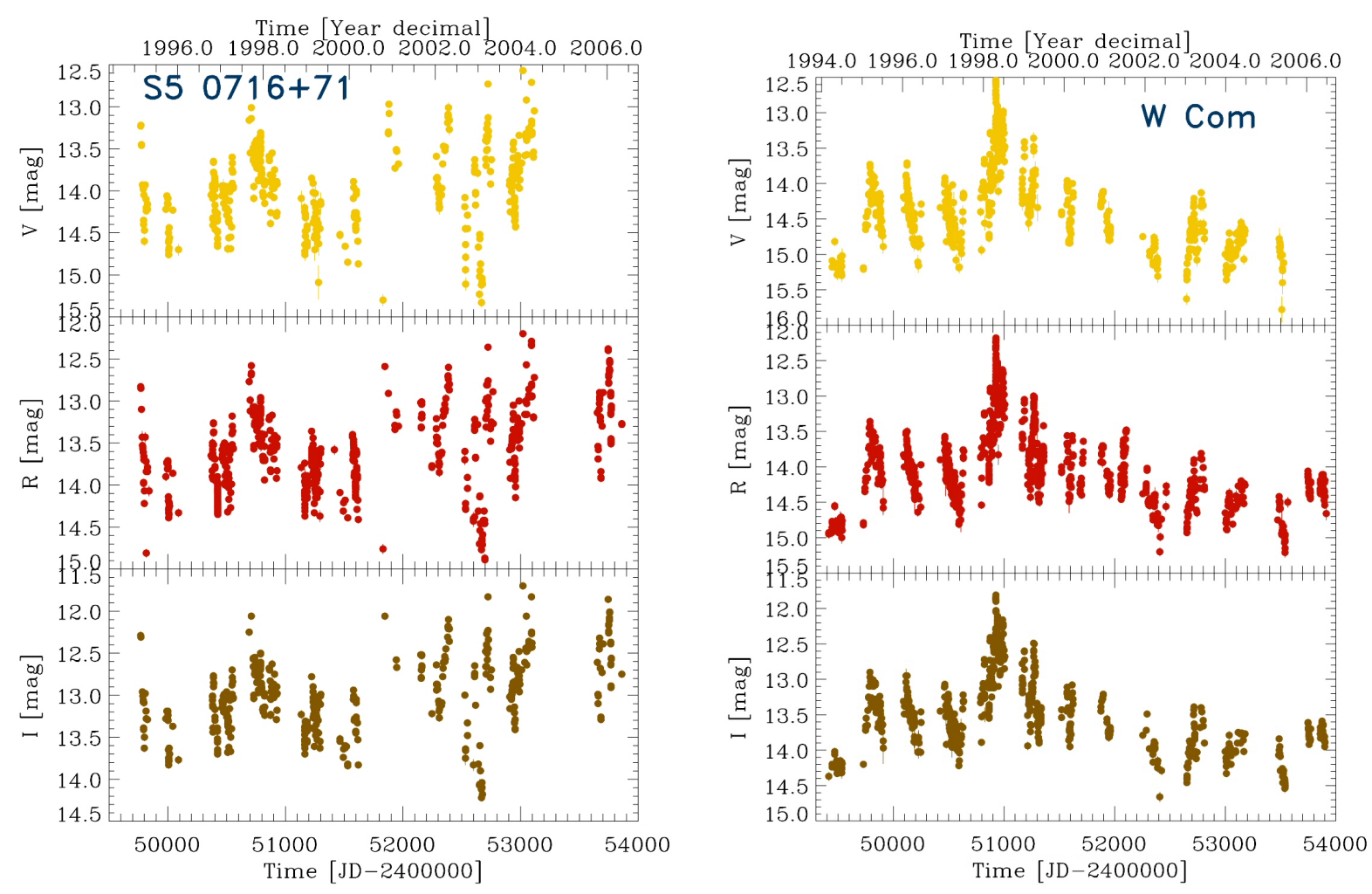

Figure 5: Optical VRI light curves of S5 0716+71 (1812 data points collected between Feb.1995 and May.2006), and of W Com (2553 data points collected between Mar.1994 and Jun.2006), obtained by the AIT.

$[5,4])$. On the other hand the power spectral density (PSD) of the variable flux time series show a shot-noise like (Brownian/brown noise) behavior. After the analysis of the continuum spectral behaviour of our whole database [6] a deep statistical temporal analysis of it is planned, while the observing monitoring is in progress.

\section{References}

[1] Carini M.T. 2004, New Astr. Rev., 48, 559

[2] Chiang J., \& Böttcher M. 2002, ApJ, 564, 92

[3] Ciprini S., Takalo L.O., Tosti G., Raiteri C.M., Fiorucci M., Villata M., Nucciarelli G., Lanteri L., Nilsson K., Ros J.A., 2007, A\&A, 467, 465

[4] Ciprini S., Tosti G., Teräsranta H., Aller, H.D. 2004, MNRAS, 348, 1379

[5] Ciprini S., Tosti G., Raiteri C.M., Villata M., Ibrahimov M.A., Nucciarelli G., Lanteri, L., 2003, A\&A, 400, 487

[6] Fiorucci M., Ciprini S., Tosti G., 2004, A\&A 419, 25

[7] Fiorucci M., Tosti G., Luciani M. 1999, MemSAIt, 70, 223

[8] Fiorucci M., \& Tosti G. 1996, A\&AS, 117, 475

[9] Fiorucci M., \& Tosti G. 1996, A\&AS, 116, 403

[10] Katajainen S., Takalo L.O., Sillanpää A., Nilsson K., Pursimo T., Hanski M., Heinämäki P., Kotoneva E., Lainela M., Nurmi P., et al. 2000, A\&AS, 143, 357 

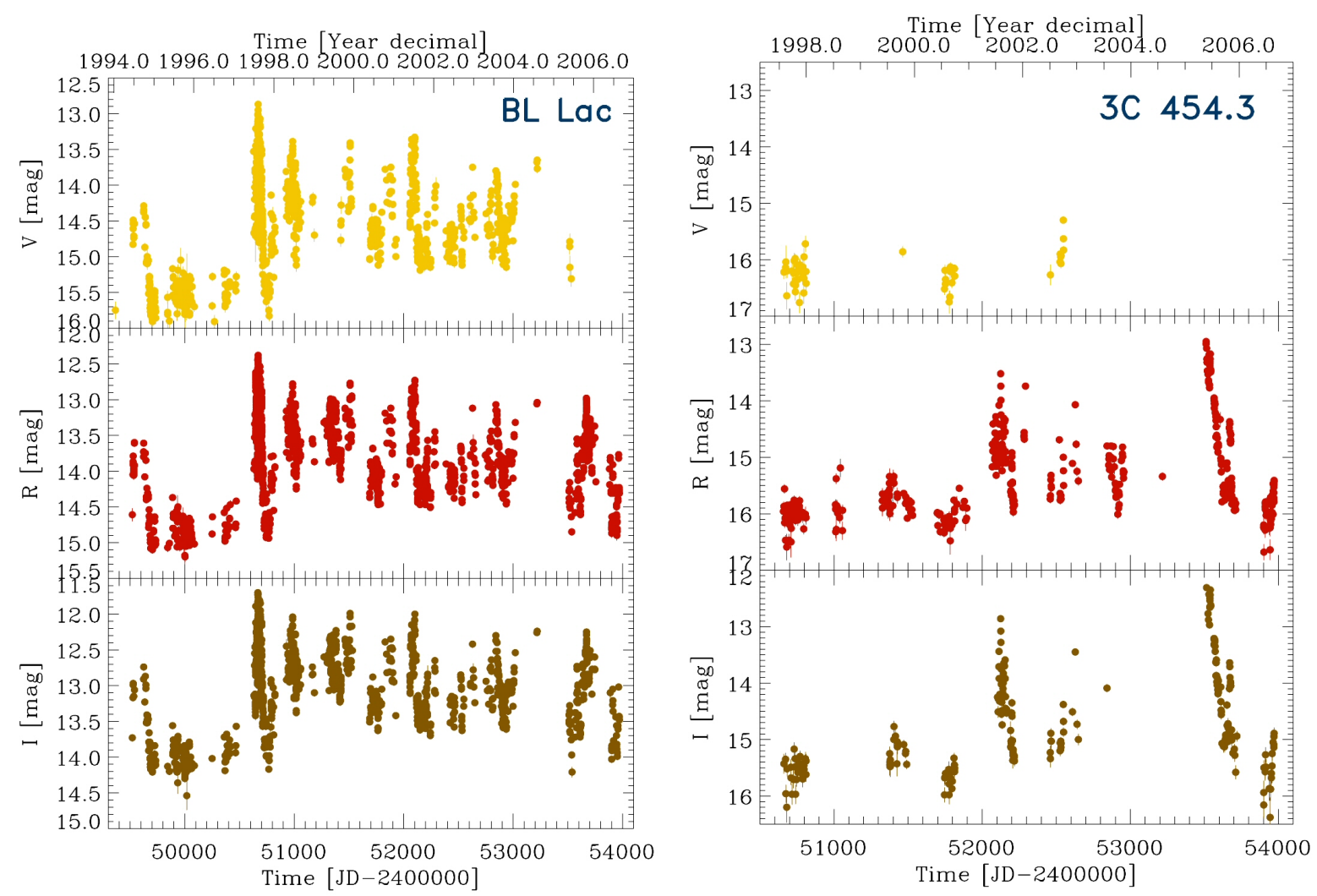

Figure 6: Optical VRI light curves of BL Lac (4545 data points collected between Jan.1994 and Aug.2006, the best observed blazar in our database), and of 3C 454.3 (737 data points collected between Jul.1997 and Aug.2006), obtained by the AIT.

[11] Massaro E., Nesci R., \& Tosti G. 2002, Ap\&SS, 282, 141

[12] Nilsson K., Pasanen M., Takalo L.O., Lindfors E., Berdyugin A., Ciprini S., Pforr J. 2007, A\&A, 475,199

[13] Spogli C., Fiorucci M., Tosti G. 1998, A\&AS, 130, 485

[14] Takalo L.O., Nilsson K., Lindfors E., Pasanen M., Berdyugin A. 2007, ASP Conf. Ser. 373, 249

[15] Tosti G., Massaro E., Nesci R., Ciprini S., Nucciarelli G., Maesano M., Montagni F., Raiteri C.M., Villata M., Lanteri L., Ostorero L., 2002, A\&A, 395, 11

[16] Tosti G., Ciprini S., Nucciarelli G. 2002, MemSAIt, 73, 1024

[17] Tosti G., Ciprini S., Massaro E., Maesano M., Montagni M., Nesci R. 2001, AIP Conf. Ser., 558, 728

[18] Tosti G., Fiorucci M., Luciani M., Rizzi N., Villata M., Raiteri C.M., de Francesco G., Lanteri L., Chiaberge M., Peila A., et al. 1998, A\&A, 130, 109

[19] Tosti G., Fiorucci M., Luciani M., Efimov Yu.S., Shakhovskoy N.M., Valtaoja E., Teräsranta H., Sillanpää A., Takalo L.O., Villata M., et al. 1998, A\&A, 339, 41

[20] Tosti G., Fiorucci M., Luciani M. 1998, ASP Conf. Ser., 159, 44

[21] Tosti G., Pascolini S., Fiorucci M., 1996, PASP, 108, 706

[22] Villata M., Raiteri C.M., Kurtanidze O.M., Nikolashvili M.G., Ibrahimov M.A., Papadakis I.E., Tsinganos K., Sadakane K., Okada N., Takalo L.O. et al. 2002, A\&A, 390, 407 